\title{
Flatland Position-Dependent-Mass: Polar Coordinates, Separability and Exact Solvability
}

\author{
S. Habib MAZHARIMOUSAVI and Omar MUSTAFA
}

Department of Physics, Eastern Mediterranean University, G Magusa, North Cyprus, Mersin 10, Turkey

E-mail: habib.mazhari@emu.edu.tr,omar.mustafa@emu.edu.tr

Received August 15, 2010, in final form October 26, 2010; Published online October 29, 2010 doi:10.3842/SIGMA.2010.088

\begin{abstract}
The kinetic energy operator with position-dependent-mass in plane polar coordinates is obtained. The separability of the corresponding Schrödinger equation is discussed. A hypothetical toy model is reported and two exactly solvable examples are studied.
\end{abstract}

Key words: position dependent mass; polar coordinates; separability; exact solvability 2010 Mathematics Subject Classification: 81Q05; 81Q60

\section{Introduction}

Position-dependent-mass (PDM) quantum particles have attracted research attention over the last few decades $[1,2,3,4,5,6,7,8,9,10,11,12,13,14,15,16,17,18,19,20,21,22,23,24,25$, 26, 27, 28, 29]. Such attention is inspired not only by the PDM feasible applicability in the study of various physical problems (e.g., many-body problem, semiconductors, quantum dots, quantum liquids, etc.), but also by the mathematical challenge associated with the corresponding von Roos Hamiltonian. The non-commutativity between the momentum operator and the positiondependent-mass introduces an ordering ambiguity in the kinetic energy operator

$$
T=-\frac{\hbar^{2}}{4}\left\{m(\vec{r})^{\gamma} \vec{\nabla} m(\vec{r})^{\beta} \cdot \vec{\nabla} m(\vec{r})^{\alpha}+m(\vec{r})^{\alpha} \vec{\nabla} m(\vec{r})^{\beta} \cdot \vec{\nabla} m(\vec{r})^{\gamma}\right\} .
$$

Obviously, changing the values of $\alpha, \beta$, and $\gamma$ would change $T$. Hence, $\alpha, \beta$, and $\gamma$ are called the ordering ambiguity parameters subjected to von Roos constraint $\alpha+\beta+\gamma=-1$ (cf., e.g., $[25,26,27,28,29])$. In the literature, one may find the orderings of Gora and William $(\beta=\gamma=0$, $\alpha=-1)$, Ben Daniel and Duke $(\alpha=\gamma=0, \beta=-1)$, Zhu and Kroemer $(\alpha=\gamma=-1 / 2, \beta=0)$, Li and Kuhn $(\beta=\gamma=-1 / 2, \alpha=0)$, and Mustafa and Mazharimousavi $(\alpha=\gamma=-1 / 4$, $\beta=-1 / 2$ ) (cf., e.g., [10, 29] for more details on this issue). However, it has been observed (cf., e.g., [29]) that the physical and/or mathematical admissibility of a given ambiguity parameters set depends not only on the continuity conditions at the abrupt heterojunction boundaries but also on the position-dependent-mass and/or potential forms. The general consensus is that there is no unique choice for these ambiguity parameters.

On the other hand, the fabrication of the essentially quasi-zero-dimensional quantum systems (like quantum dots (QD)) that are populated by two dimensional flatland quantum particles (electrons in the QDs case) confined by an artificial potential has inspired intensive research activities. Under such dimensional settings, a quantum particle endowed with a positiondependent-mass, $m(\vec{r})=m_{\circ} M(\vec{r})=m_{\circ} M(\rho, \varphi)$, would be an interesting case to study, therefore. To the best of our knowledge, such PDM settings have never been discussed in the literature, within our forthcoming methodical proposal at least.

For the sake of separability, we recollect (in Section 2) the essentials of the kinetic energy operator in plane polar coordinates mandated by a position dependent mass of the form 
$M(\rho, \varphi)=g(\rho) f(\varphi), g(\rho)=\rho^{-2}$ and an interaction potential $V(\rho, \varphi)=\tilde{V}(\rho) / f(\varphi)$. In Section 3, we show that whilst the radial part (in (7) below) can be brought into the one-dimensional Schrödinger format through a simple choice $R(\rho)=\rho^{-3 / 2} U(\rho)$, a point canonical transformation (PCT) is needed for the azimuthal angular part (see (8) below). Moreover, a hypothetical toy model with $M(\rho, \varphi)=\rho^{-2} \cos ^{2} \varphi, \rho \in(0, \infty), \varphi \in(0,2 \pi)$, and $V(\rho, \varphi)=-V_{\circ} \rho^{2 k} / 2 \cos ^{2} \varphi$, $V_{\circ}>0$, is considered (in the same section). Two constructive exactly-solvable toy examples of fundamental nature, with $m(\rho, \varphi)=1 / \rho^{2}$, are given in Section 4. Namely and effectively, a Coulomb-like and a harmonic-oscillator-like radial Schrödinger models. We conclude in Section 5.

\section{Essentials of the kinetic energy operator in plane polar coordinates and separability}

The kinetic energy operator $T$, in (1), for a PDM quantum particle with $m(\vec{r})=M(\rho, \varphi)$, moving in a two-dimensional flatland (with $\hbar=m_{\circ}=1$ units), reads

$$
T=-\frac{1}{4}\left\{M(\rho, \varphi)^{\gamma} \vec{\nabla} M(\rho, \varphi)^{\beta} \cdot \vec{\nabla} M(\rho, \varphi)^{\alpha}+M(\rho, \varphi)^{\alpha} \vec{\nabla} M(\rho, \varphi)^{\beta} \cdot \vec{\nabla} M(\rho, \varphi)^{\gamma}\right\} .
$$

This would, with $M(\rho, \varphi) \equiv M$ (for economy of notation) and

$$
\vec{\nabla}=\hat{\rho} \partial_{\rho}+\hat{\varphi} \frac{1}{\rho} \partial_{\varphi} \quad \Longrightarrow \quad \vec{\nabla} \cdot \vec{\nabla}=\nabla^{2}=\frac{1}{\rho} \partial_{\rho}\left(\rho \partial_{\rho}\right)+\frac{1}{\rho^{2}} \partial_{\varphi}^{2}
$$

yield (in a straightforward though rather tedious manner)

$$
T=-\frac{1}{2}\left[2 W(\rho, \varphi)+\left(\frac{1}{\rho M}-\frac{M_{\rho}}{M^{2}}\right) \partial_{\rho}-\frac{M_{\varphi}}{\rho^{2} M^{2}} \partial_{\varphi}+\frac{1}{M} \partial_{\rho}^{2}+\frac{1}{\rho^{2} M} \partial_{\varphi}^{2}\right],
$$

where

$$
\begin{aligned}
& W(\rho, \varphi)=\frac{1}{4}\left[\frac{\xi}{M^{3}}\left(M_{\rho}^{2}+\frac{M_{\varphi}^{2}}{\rho^{2}}\right)+\frac{(\alpha+\gamma)}{M^{2}}\left(\frac{M_{\rho}}{\rho}+M_{\rho \rho}+\frac{M_{\varphi \varphi}}{\rho^{2}}\right)\right], \\
& \xi=\alpha(\alpha-1)+\gamma(\gamma-1)-\beta(\beta+1),
\end{aligned}
$$

and

$$
M_{\rho}=\partial_{\rho} M(\rho, \varphi), \quad M_{\rho \rho}=\partial_{\rho}^{2} M(\rho, \varphi), \quad M_{\varphi}=\partial_{\varphi} M(\rho, \varphi), \quad M_{\varphi \varphi}=\partial_{\varphi}^{2} M(\rho, \varphi) .
$$

A recollection of the time-independent Schrödinger equation, for the PDM quantum particle with $m(\vec{r})=M(\rho, \varphi)$ moving under the influence of a two-dimensional flatland potential $V(\rho, \varphi)$, implies that

$$
T \psi(\rho, \varphi)+V(\rho, \varphi) \psi(\rho, \varphi)=E \psi(\rho, \varphi)
$$

where $T$ is given in (2). The separation of variables of which suggests a wave function of the form

$$
\psi(\rho, \varphi)=R(\rho) \Phi(\varphi), \quad \rho \in(0, \infty), \quad \varphi \in(0,2 \pi),
$$

to obtain

$$
\left(\frac{1}{\rho M}-\frac{M_{\rho}}{M^{2}}\right) \frac{\partial_{\rho} R}{R}-\frac{M_{\varphi}}{\rho^{2} M^{2}} \frac{\partial_{\varphi} \Phi}{\Phi}+\frac{1}{M} \frac{\partial_{\rho}^{2} R}{R}+\frac{1}{\rho^{2} M} \frac{\partial_{\varphi}^{2} \Phi}{\Phi}=-2[E-V(\rho, \varphi)+W(\rho, \varphi)] .
$$


In the search of feasible separability of this equation, it is obvious that a mass setting of the form

$$
M(\rho, \varphi)=g(\rho) f(\varphi)
$$

would, with

$$
g(\rho)=\rho^{-2},
$$

ease separability and allow $W(\rho, \varphi) \longrightarrow \tilde{W}(\varphi)$, where

$$
\tilde{W}(\varphi)=\frac{1}{4}\left[\xi\left(\frac{4}{f(\varphi)}+\frac{\left[\partial_{\varphi} f(\varphi)\right]^{2}}{f(\varphi)^{3}}\right)+\frac{(\alpha+\gamma)}{f(\varphi)}\left(4+\frac{\partial_{\varphi}^{2} f(\varphi)}{f(\varphi)}\right)\right] .
$$

Moreover, if the position-dependent-mass $M(\rho, \varphi)$ in (4) is interrelated with the interaction potential $V(\rho, \varphi)$ through $f(\varphi)$ of $(4)$ so that

$$
V(\rho, \varphi)=\frac{\tilde{V}(\rho)}{f(\varphi)}
$$

one may recast equation (3) as

$$
3 \rho \frac{\partial_{\rho} R}{R}+\rho^{2} \frac{\partial_{\rho}^{2} R}{R}-2 \tilde{V}(\rho)=\frac{\partial_{\varphi} f(\varphi)}{f(\varphi)} \frac{\partial_{\varphi} \Phi}{\Phi}-\frac{\partial_{\varphi}^{2} \Phi}{\Phi}-2 f(\varphi)[E+\tilde{W}(\varphi)]=\lambda,
$$

where $\lambda$ is the separation constant. The separability of (6) is obvious, therefore. That is, the radial equation reads

$$
\left[\rho^{2} \partial_{\rho}^{2}+3 \rho \partial_{\rho}-2 \tilde{V}(\rho)\right] R(\rho)=\lambda R(\rho)
$$

and the azimuthal angular equation reads

$$
\left\{-\partial_{\varphi}^{2}+\frac{\partial_{\varphi} f(\varphi)}{f(\varphi)} \partial_{\varphi}-2 f(\varphi)[E+\tilde{W}(\varphi)]\right\} \Phi(\varphi)=\lambda \Phi(\varphi)
$$

\section{Corresponding one-dimensional Schrödinger equations}

It is obvious that, the substitution $R(\rho)=\rho^{j} U(\rho), j=-3 / 2$, would eliminate the first-order derivative in equation (7) to obtain

$$
\left\{-\partial_{\rho}^{2}+\left[\frac{(3 / 4+\lambda)}{\rho^{2}}+\frac{2 \tilde{V}(\rho)}{\rho^{2}}\right]\right\} U(\rho)=0, \quad \rho \in(0, \infty) .
$$

Which suggests that the effective radial potential is

$$
\tilde{V}_{\text {eff }}(\rho)=\frac{(3 / 4+\lambda)}{\rho^{2}}+\frac{2 \tilde{V}(\rho)}{\rho^{2}} .
$$

On the other hand, a point canonical transformation

$$
q^{\prime}(\varphi)=\partial_{\varphi} q(\varphi)=\sqrt{f(\varphi)}
$$

with the substitution

$$
\Phi(\varphi)=f(\varphi)^{1 / 4} \chi(q(\varphi))
$$


would transform the azimuthal angular equation (8) into

$$
-\frac{1}{2} \chi^{\prime \prime}(q)+W_{\text {eff }}(q) \chi(q)=E \chi(q)
$$

where

$$
\begin{aligned}
W_{\mathrm{eff}}(q) & =-\tilde{W}(\varphi)+\frac{7\left[\partial_{\varphi} f(\varphi)\right]^{2}}{32 f(\varphi)^{3}}-\frac{\partial_{\varphi}^{2} f(\varphi)}{8 f(\varphi)^{2}}-\frac{\lambda}{2 f(\varphi)} \\
& =\frac{\left[\partial_{\varphi} f(\varphi)\right]^{2}}{32 f(\varphi)^{3}}(7-8 \xi)-\frac{\partial_{\varphi}^{2} f(\varphi)}{8 f(\varphi)^{2}}(1+2(\alpha+\gamma))-\frac{1}{f(\varphi)}\left(\xi+\alpha+\gamma+\frac{\lambda}{2}\right) .
\end{aligned}
$$

Few illustrative toy examples are in order.

\subsection{A hypothetical toy model}

In this toy model we choose to work on a quantum particle endowed with a position dependent mass

$$
M(\rho, \varphi)=\rho^{-2} \cos ^{2} \varphi, \quad \rho \in(0, \infty), \quad \varphi \in(0,2 \pi),
$$

under the influence of

$$
V(\rho, \varphi)=-V_{\circ} \frac{\rho^{2 k}}{2 \cos ^{2} \varphi}, \quad V_{\circ}>0 .
$$

This would effectively mean that $g(\rho)=\rho^{-2}, f(\varphi)=\cos ^{2} \varphi$, and $\tilde{V}(\rho)=-V_{\circ} \rho^{2 k} / 2$. Although such a toy model may not relate to any practical importance, it could be theoretically and/or mathematically appealing.

To deal with the radial part in equation (9), we may recollect that the Bessel's equation $\rho^{2} Z_{n}^{\prime \prime}(\rho)+\rho Z_{n}^{\prime}(\rho)+\left(\rho^{2}-n^{2}\right) Z_{n}(\rho)=0$ with $Z_{n}(\rho)=U_{n}(\rho) / \sqrt{\rho}$ collapses into

$$
\left\{\partial_{\rho}^{2}+\left[1+\frac{\left(1-4 n^{2}\right)}{4 \rho^{2}}\right]\right\} U(\rho)=0 .
$$

Which, when compared with (9), suggests that $V_{\circ}=1, k=1$, and $\lambda=n^{2}-1$ are feasibly admissible parametric settings for a physically acceptable well-behaved solution in the form of Bessel functions, i.e.,

$$
U_{n}(\rho)=A \sqrt{\rho} J_{n}(\rho) \quad \Rightarrow \quad R_{n}(\rho)=\frac{A}{\rho} J_{n}(\rho) .
$$

On the other hand, the effective potential (11) of the azimuthal angular part of (10), with $q^{\prime}(\varphi)=\sqrt{f(\varphi)} \Longrightarrow q(\varphi)=\sin \varphi$, reads

$$
W_{\text {eff }}(q)=\frac{\zeta_{1} \sin ^{2} \varphi-\zeta_{2}}{\cos ^{4} \varphi}=\frac{\zeta_{1} q^{2}-\zeta_{2}}{\left(1-q^{2}\right)^{2}},
$$

where

$$
\zeta_{1}=\frac{3}{8}+\frac{\lambda}{2}
$$

and

$$
\zeta_{2}=\frac{\lambda}{2}-\frac{1}{4}+\alpha\left(\alpha-\frac{1}{2}\right)+\gamma\left(\gamma-\frac{1}{2}\right)-\beta(\beta+1) .
$$


At this point, one may wish to set $W_{\text {eff }}(q)=0$ by requiring $\zeta_{1}=0=\zeta_{2}$. This would yield that $\lambda=-3 / 4$ (hence $n=1 / 2$ ). Introducing (in addition to von Roos constraint $\alpha+\beta+\gamma=-1$ ) yet another constraint on the ordering ambiguity parameters, therefore. That is,

$$
\frac{5}{8}=\alpha\left(\alpha-\frac{1}{2}\right)+\gamma\left(\gamma-\frac{1}{2}\right)-\beta(\beta+1) .
$$

In this case, the azimuthal angular part of the wave function would, with $\chi(q(\varphi))=\exp (i m q)=$ $\exp (i m \sin \varphi)$, read

$$
\Phi(\varphi)=f(\varphi)^{1 / 4} \chi(q(\varphi))=\sqrt{\cos \varphi} e^{i m \sin \varphi},
$$

where the energy eigenvalues are given as $E=E_{m}=m^{2} / 2(m=0, \pm 1, \pm 2, \ldots$ is the magnetic quantum number). Hereby, we notice that the only available quantum state is the one with an irrational radial quantum number $n=1 / 2$ (which can be thought of as $n=n_{\rho}+1 / 2$, where $n_{\rho}$ is the regular radial quantum number, $n_{\rho}=0$ in this particular case). Hence, the radial wave function is $R(\rho)=C J_{1 / 2}(\rho) / \rho$. Moreover, it should be noted that the constraint in (14) is satisfied when $\alpha=\gamma=-1 / 4, \beta=-1 / 2$ (i.e., Mustafa's and Mazharimousavi's ordering [10]). This should not necessarily mean that the other orderings available in the literature are no good (cf., e.g., [10]).

However, should we choose $\zeta_{1} \neq 0 \neq \zeta_{2}$ in $W_{\text {eff }}(q)$ of (12), equation (10) would then admit a solution in terms of Heun Confluent functions, HeunC, as

$$
\begin{aligned}
\Phi(\varphi)= & f(\varphi)^{1 / 4} \chi(q(\varphi))=\sqrt{\cos \varphi}\left[(i \cos \varphi)^{1+\sqrt{2 \zeta_{1}-2 \zeta_{2}+1}}\right] \\
& \times\left\{C_{1} \operatorname{HeunC}\left(0,-\frac{1}{2}, \sqrt{2 \zeta_{1}-2 \zeta_{2}+1}, \frac{E}{2},-\frac{E}{2}-\frac{\zeta_{2}}{2}+\frac{1}{2}, \sin ^{2} \varphi\right)\right. \\
& \left.+C_{2} \sin \varphi \operatorname{HeunC}\left(0, \frac{1}{2}, \sqrt{2 \zeta_{1}-2 \zeta_{2}+1}, \frac{E}{2},-\frac{E}{2}-\frac{\zeta_{2}}{2}+\frac{1}{2}, \sin ^{2} \varphi\right)\right\},
\end{aligned}
$$

Which is to be subjected to the boundary conditions $\Phi(\varphi)=\Phi(\varphi+2 \pi)$, indeed. Here, $C_{1}$ and $C_{2}$ are constants to be determined. Under such settings, moreover, it is obvious that one should choose a value of $E$ (consequently $\zeta_{2}$ and $\zeta_{1}$ would be determined for specific values of the ordering ambiguity parameters) to find some value for $\lambda$. Nevertheless, labeling/classifying the quantum states (i.e., $1 s, 2 s, 2 p, \ldots$, etc.) seems to be non-negotiable and lost in the jam of the multi-parametric settings, manifested by not only the chosen position dependent mass and potential but also by the ordering ambiguity parameters as well. However, such experimental toy model inspires the exploration of the forthcoming interesting toy examples.

\section{Constructive, exactly-solvable two toy examples with $m(\rho, \varphi)=1 / \rho^{2}$}

In this section, we consider two examples of exactly-solvable nature (many other models do amply exist). Strictly speaking, a quantum particle endowed with a position dependent mass with $g(\rho)=1 / \rho^{2}$ and $f(\varphi)=1$ (hence $M(\rho, \varphi)=1 / \rho^{2}$ ) would, in effect, collapse equation (5) into

$$
\tilde{W}(\varphi)=\alpha^{2}+\gamma^{2}-\beta(\beta+1),
$$

and simplify equation (8) to read

$$
\left\{-\partial_{\varphi}^{2}-[2 E+2 \tilde{W}(\varphi)+\lambda]\right\} \Phi(\varphi)=0,
$$


where $\Phi(\varphi)$ should be a single-valued function (i.e., $\Phi(\varphi)=\Phi(\varphi+2 \pi)$ ). Therefore, it takes the form $\Phi(\varphi)=e^{i m \varphi}$. In this case, $2 E+2 \tilde{W}(\varphi)+\lambda=m^{2}$ and

$$
E=\frac{m^{2}-\lambda}{2}-\left[\alpha^{2}+\gamma^{2}-\beta(\beta+1)\right]
$$

Moreover, we now consider that such a PDM quantum particle is moving in a force field described through

$$
\tilde{V}(\rho)=\frac{1}{2} \omega^{2} \rho^{2}-\rho .
$$

One would then recast equation (9) as

$$
\left\{-\partial_{\rho}^{2}+\left[\frac{\ell^{2}-1 / 4}{\rho^{2}}+\omega^{2}-\frac{2}{\rho}\right]\right\} U(\rho)=0
$$

where $\ell=|m|$ and $\ell^{2}-1 / 4=3 / 4+\lambda \Longrightarrow \ell=(\lambda+1)^{1 / 2}$. Hereby, it should be noted that equation (16) is a two-dimensional Coulombic-like Schrödinger equation. As such, its solution can be inferred from the well-known two-dimensional Coulombic one. The eigenvalues of which read

$$
\omega^{2}=\left(n_{\rho}+\ell+1\right)^{-2} \quad \Longrightarrow \quad \omega=\left(n_{\rho}+\ell+1\right)^{-1}=\left(n_{\rho}+\sqrt{\lambda+1}+1\right)^{-1},
$$

and yield (with $\omega=1 / b$ for simplicity)

$$
\lambda=\left(b-n_{\rho}-1\right)^{2}-1,
$$

where $n_{\rho}=0,1,2, \ldots$ is the radial quantum number. In this case, equation (15) reads the energy eigenvalues as

$$
E_{n_{\rho}, m}=\frac{1}{2}\left[m^{2}-\left(b-n_{\rho}-1\right)^{2}+1\right]-\left[\alpha^{2}+\gamma^{2}-\beta(\beta+1)\right] .
$$

Next, we consider the same PDM quantum particle moving now in a radial potential (replacing that in (16), in effect) described by

$$
\tilde{V}(\rho)=\frac{a^{2}}{8} \rho^{4}-\frac{d}{2} \rho^{2}
$$

Under such setting, equation (9) reads

$$
\left\{-\partial_{\rho}^{2}+\left[\frac{\ell^{2}-1 / 4}{\rho^{2}}+\frac{a^{2}}{4} \rho^{2}-d\right]\right\} U(\rho)=0,
$$

which is effectively a two-dimensional harmonic-oscillator-like problem that admits eigenvalues of the form

$$
d=a\left(2 n_{\rho}+\sqrt{\lambda+1}+1\right) \quad \Longrightarrow \quad \lambda=\left(\frac{d}{a}-2 n_{\rho}-1\right)^{2}-1 .
$$

Therefore, the energy eigenvalues for such an oscillator-like problem are

$$
E_{n_{\rho}, m}=\frac{1}{2}\left[m^{2}-\left(\frac{d}{a}-2 n_{\rho}-1\right)^{2}+1\right]-\left[\alpha^{2}+\gamma^{2}-\beta(\beta+1)\right] .
$$

Obviously, the degeneracies associated with the magnetic quantum number $(m=0, \pm 1, \pm 2, \ldots)$ in the energies (17) and (18) are unavoidably in point. To remove such degeneracies, a magnetic field applied perpendicular to the current two dimensional flatland settings (through the symmetric gauge $\mathbf{A}=\mathbf{B}(-y, x, 0) / 2$ that may introduce a term $\sim m B$ to be added to $\lambda$ (cf., e.g., Mustafa [30]) could be sought as a remedy. However, such a study readily lies far beyond our current methodical proposal. Yet, additional degeneracies associated with the ordering ambiguity parameters $\alpha$ and $\gamma$ (but not $\beta$ ) are observed. Moreover, within similar parametric changes, one can also get the corresponding wave functions. 


\section{Concluding remarks}

The kinetic energy operator for a quantum particle endowed with a position dependent mass is a problem with many aspects that are yet to be explored. In the current work, we tried to study this problem within the context of the flatland plane-polar coordinates $(\rho, \varphi)$. In due course, the essentials of the kinetic energy operator in plane-polar coordinates are reported. The separability of the related Schrödinger equation is sought through a position dependent mass $M(\rho, \varphi)=g(\rho) f(\varphi)$, accompanied by an interaction potential $V(\rho, \varphi)=\tilde{V}(\rho) / f(\varphi)$. Such a combination is not a unique one.

In the light of our experience above, some observations are in order.

Apart from its practical applicability and multi-parametric complexity sides, the hypothetical toy model $\left(M(\rho, \varphi)=\rho^{-2} \cos ^{2} \varphi\right.$ and $\left.V(\rho, \varphi)=-V_{\circ} \rho^{2 k} / 2 \cos ^{2} \varphi\right)$ has provided a road-map on the possible technical difficulties. We have observed that for a physically acceptable radial solution, for (9), one may consider $V_{\circ}=1, k=1$, and $\lambda=n^{2}-1$ as proper parametric settings. Moreover, for the azimuthal angular part with $\zeta_{1}=0=\zeta_{2}$ in (12), we have found that there is only one quantum state with a radial quantum number $n_{\rho}=0(n=1 / 2)$ and a corresponding magnetic quantum number $m=0, \pm 1, \pm 2, \ldots$ However, for $\zeta_{1} \neq 0 \neq \zeta_{2}$, the problem becomes more complicated and one may solve it following the simplest possible way. For example, instead of choosing $\lambda$ of (13) and calculate $E$ of (10), one may choose a value for $E$ first and calculate $\lambda$ (a process that is contemplated to indulge some graphical estimations). Indeed, the quantum states are readily there but we were unable to properly identify/classify them (within the context of the well known quantum numbers). Such a "quasi-quantum-miss", say, did not repeat itself for the rest of the examples reported above (i.e., the quantum states were very well identified/classified within the known quantum numbers as documented in Section 4).

Moreover, we have shown that for a quantum particle with $m(\rho, \varphi)=1 / \rho^{2}$ moving in a potential $\tilde{V}(\rho)=\omega^{2} \rho^{2} / 2-\rho$, the corresponding Schrödinger equation is transformed into an effectively radial Coulombic problem. Yet, if the same PDM particle is moving in $\tilde{V}(\rho)=a^{2} \rho^{4} / 8-d \rho^{2} / 2$, then the Schrödinger equation collapses into a radial harmonic-oscillator problem. In both cases, the exact solutions can be inferred from the known textbook ones. One observes obvious degeneracies associated not only with the magnetic quantum number $m$ but also associated with the ordering ambiguity parameters $\alpha$ and $\gamma$ (but not $\beta$ ).

Finally, it should be noted that the applicability of the attendant methodical proposal can be extended to deal with non-hermitian Hamiltonians as well (cf., e.g., [28] and related references therein).

\section{References}

[1] Puente A., Casas M., Non-local energy density functional for atoms and metal clusters, Comput. Mater Sci. 2 (1994), 441-449.

[2] Plastino A.R., Casas M., Plastino A., Bohmian quantum theory of motion for particles with positiondependent effective mass, Phys. Lett. A 281 (2001), 297-304.

[3] Schmidt A.G.M., Wave-packet revival for the Schrödinger equation with position-dependent mass, Phys. Lett. A 353 (2006), 459-462.

[4] Dong S.H., Lozada-Cassou M., Exact solutions of the Schrödinger equation with the position-dependent mass for a hard-core potential, Phys. Lett. A 337 (2005), 313-320.

[5] Vakarchuk I.O., The Kepler problem in Dirac theory for a particle with position-dependent mass, J. Phys. A: Math. Gen. 38 (2005), 4727-4734, quant-ph/0502105.

[6] Cai C.-Y., Ren Z.-Z., Ju G.-X., Exact solutions to three-dimensional Schrödinger equation with an exponentially position-dependent mass, Commun. Theor. Phys. (Beijing) 43 (2005), 1019-1022.

[7] Roy B., Roy P., Effective mass Schrödinger equation and nonlinear algebras, Phys. Lett. A 340 (2005), 70-73. 
[8] Gönül B., Koçak M., Remarks on exact solvability of quantum systems with spatially varying effective mass, Chinese Phys. Lett. 20 (2005), 2742-2745.

[9] de Souza Dutra A., Almeida C.A.S., Exact solvability of potentials with spatially dependent effective masses, Phys Lett. A 275 (2000), 25-30.

[10] Mustafa O., Mazharimousavi S.H., Ordering ambiguity revisited via position dependent mass pseudomomentum operators, Internat. J. Theoret. Phys. 46 (2007), 1786-1796, quant-ph/0607158.

[11] Cruz y Cruz S., Negro J., Nieto L.M., Classical and quantum position-dependent mass harmonic oscillators, Phys. Lett. A 369 (2007), 400-406.

[12] Cruz y Cruz S., Rosas-Ortiz O., Position-dependent mass oscillators and coherent states, J. Phys. A: Math. Theor. 42 (2009), 185205, 21 pages.

[13] Lekner J., Reflectionless eigenstates of the sech ${ }^{2}$ potential, Amer. J. Phys. 75 (2007), 1151-1157.

[14] Quesne C., Tkachuk V.M., Deformed algebras, position-dependent effective masses and curved spaces: an exactly solvable Coulomb problem, J. Phys. A: Math. Gen. 37 (2004), 4267-4281, math-ph/0403047.

[15] Jiang L., Yi L.-Z., Jia C.-S., Exact solutions of the Schrödinger equation with position-dependent mass for some Hermitian and non-Hermitian potentials, Phys. Lett. A 345 (2005), 279-286.

[16] Mustafa O., Mazharimousavi S.H., Quantum particles trapped in a position-dependent mass barrier: a $d$ dimensional recipe, Phys. Lett. A 358 (2006), 259-261, quant-ph/0603134.

[17] Diaz J.I., Negro J., Nieto L.M., Rosas-Ortiz O., The supersymmetric modified Pöschl-Teller and delta well potentials, J. Phys. A: Math. Gen. 32 (1999), 8447-8460, quant-ph/9910017.

[18] Alhaidari A.D., Solutions of the nonrelativistic wave equation with position-dependent effective mass, Phys. Rev. A 66 (2002), 042116, 7 pages, quant-ph/0207061.

Gritsev V.V., Kurochkin Y.A., Model of excitations in quantum dots based on quantum mechanics in spaces of constant curvature, Phys. Rev. B 64 (2001), 035308, 9 pages.

[19] Mustafa O., Mazharimousavi S.H., $d$-dimensional generalization of the point canonical transformation for a quantum particle with position-dependent mass, J. Phys. A: Math. Gen. 39 (2006), 10537-10547, math-ph/0602044.

Lévai G., Özer O., An exactly solvable Schrödinger equation with finite positive position-dependent effective mass, J. Math. Phys. 51 (2010), 092103, 13 pages.

[20] Bagchi B., Banerjee A., Quesne C., Tkachuk V.M., Deformed shape invariance and exactly solvable Hamiltonians with position-dependent effective mass, J. Phys. A: Math. Gen. 38 (2005), 2929-2945, quant-ph/0412016.

Bagchi B., Ganguly A., Sinha A., Supersymmetry across nanoscale heterojunction, Phys. Lett. A 374 (2010), 2397-2400, arXiv:1002.2732.

[21] Yu J., Dong S.-H., Exactly solvable potentials for the Schrödinger equation with spatially dependent mass, Phys. Lett. A 325 (2004), 194-198.

[22] Quesne C., First-order intertwining operators and position-dependent mass Schrödinger equations in $d$ dimensions, Ann. Physics 321 (2006), 1221-1239, quant-ph/0508216.

[23] Tanaka T., $N$-fold supersymmetry in quantum systems with position-dependent mass, J. Phys. A: Math. Gen. 39 (2006), 219-234, quant-ph/0509132.

[24] de Souza Dutra A., Ordering ambiguity versus representation, J. Phys. A: Math. Gen. 39 (2006), 203-208, arXiv:0705.3247.

[25] von Roos O., Position-dependent effective masses in semiconductor theory, Phys. Rev. B 27 (1983), $7547-$ 7552.

Lévy-Leblond J.M., Position-dependent effective mass and Galilean invariance, Phys. Rev. A 52 (1995), 1845-1849.

[26] Mustafa O., Mazharimousavi S.H., Non-Hermitian $d$-dimensional Hamiltonians with position-dependent mass and their $\eta$-pseudo-Hermiticity generators, Czechoslovak J. Phys. $56 \quad$ (2006), 967-975, quant-ph/0603272.

[27] Mustafa O., Mazharimousavi S.H., $\eta$-weak-pseudo-Hermiticity generators and exact solvability, Phys. Lett. A 357 (2006), 295-297, quant-ph/0604106.

[28] Mustafa O., Mazharimousavi S.H., Complexified von Roos Hamiltonian's $\eta$-weak-pseudo-Hermiticity, isospectrality and exact solvability, J. Phys. A: Math. Theor. 41 (2008), 244020, 8 pages, arXiv:0707.3738.

[29] Mustafa O., Mazharimousavi S.H., A singular position-dependent mass particle in an infinite potential well, Phys. Lett. A 373 (2009), 325-327, arXiv:0807.3030.

[30] Mustafa O., The shifted-1/N-expansion method for two-dimensional hydrogenic donor states in an arbitrary magnetic field, J. Phys.: Condens. Matter 5 (1993), 1327-1332. 